\title{
Design and Evaluation of an Acceleration Guidance Algorithm for Entry
}

\author{
A. Saraf, ${ }^{*}$ J. A. Leavitt, ${ }^{\dagger}$ D. T. Chen,${ }^{\ddagger}$ and K. D. Mease ${ }^{\S}$ \\ University of California, Irvine, Irvine, California 92697
}

\begin{abstract}
The design and performance evaluation of an entry guidance algorithm for future space transportation vehicles is presented. The guidance concept is to plan and track aerodynamic acceleration. This concept, on which the longitudinal entry guidance for the Space Shuttle Orbiter is based, is extended to integrated longitudinal and lateral guidance. With integrated longitudinal and lateral guidance, more extreme points in the landing footprint can be reached accurately; in particular, the cross-range capability is extended. The guidance algorithm consists of two components: a trajectory planner and a trajectory tracking law. The planner generates reference drag acceleration and heading angle profiles, along with reference state and bank angle profiles. The planner executes onboard and is capable of generating updates as the entry evolves. The tracking law, based on feedback linearization, commands the angles of bank and attack required to follow the reference drag and heading angle profiles. The planner and tracking law are described, along with additional higher level logic included in the algorithm. Extensive simulations for a set of return-from-orbit entries, including ones requiring large cross range, demonstrate that this algorithm consistently achieves the desired target conditions within allowable tolerances and satisfies all other entry constraints.
\end{abstract}

\section{Nomenclature}

$A=$ reference wing area, $\mathrm{ft}^{2}$

$a_{\max }=$ maximum allowable normal acceleration, $\mathrm{ft} / \mathrm{s}^{2}$

$a_{n}=$ normal acceleration, $\mathrm{ft} / \mathrm{s}^{2}$

$C_{D}=$ coefficient of drag

$C_{L}=$ coefficient of lift

$C_{\gamma}=$ Coriolis acceleration term in $\gamma^{\prime}$ equation, $\mathrm{rad} \cdot \mathrm{s}^{2} / \mathrm{ft}^{2}$

$C_{\psi}=$ Coriolis acceleration term in $\psi^{\prime}$ equation, $\mathrm{rad} \cdot \mathrm{s}^{2} / \mathrm{ft}^{2}$

$D=$ drag acceleration, $\mathrm{ft} / \mathrm{s}^{2}$

$E=$ energy divided by vehicle mass, $\mathrm{ft}^{2} / \mathrm{s}^{2}$

$\tilde{E}=$ normalized energy

$g=$ gravitational acceleration, $\mathrm{ft} / \mathrm{s}^{2}$

$h_{s}=$ scale height, $\mathrm{ft}$

$k_{1}=$ integral gain in drag tracking law

$k_{2}=$ proportional gain in heading angle tracking law

$k_{3}=$ integral gain in heading angle tracking law

$L=$ lift acceleration, $\mathrm{ft} / \mathrm{s}^{2}$

$M=$ Mach number

$m \quad=$ vehicle mass, slugs

$\bar{q}=$ dynamic pressure, $1 \mathrm{bf} / \mathrm{ft}^{2}$

$\dot{q}=$ heat rate, $\mathrm{Btu} /\left(\mathrm{ft}^{2} \cdot \mathrm{s}\right)$

$R=$ downrange, $\mathrm{ft}$

$r \quad=$ radial distance from vehicle to planet center, $\mathrm{ft}$

$S \quad=$ trajectory length, $\mathrm{ft}$

$u_{D}=$ pseudocontrol in drag dynamics

$u_{\psi}=$ pseudocontrol in heading angle dynamics

Received 10 February 2003; revision received 19 September 2003; accepted for publication 24 September 2003. Copyright (C) 2003 by the authors. Published by the American Institute of Aeronautics and Astronautics, Inc., with permission. Copies of this paper may be made for personal or internal use, on condition that the copier pay the $\$ 10.00$ per-copy fee to the Copyright Clearance Center, Inc., 222 Rosewood Drive, Danvers, MA 01923; include the code 0022-4650/04 \$10.00 in correspondence with the CCC.

*Postdoctoral Researcher, Department of Mechanical and Aerospace Engineering; asaraf@uci.edu. Member AIAA.

${ }^{\dagger}$ Graduate Research Assistant, Department of Mechanical and Aerospace Engineering; jleavitt@uci.edu.

Fraduate Research Assistant, Department of Mechanical and Aerospace Engineering; currently Flight Systems Engineer, Universal Space Lines, LLC, 1501 Quail Street, Suite 100, Newport Beach, CA 92660-2726; dtchen@spacelines.com.

${ }^{\S}$ Professor, Department of Mechanical and Aerospace Engineering; kmease@uci.edu. Associate Fellow AIAA.
$V \quad=$ planet-relative speed, $\mathrm{ft} / \mathrm{s}$

$\alpha=$ angle of attack, rad

$\gamma \quad=$ flight-path angle, rad

$\zeta=$ damping ratio of desired drag error dynamics

$\theta=$ longitude, $\mathrm{rad}$

$\mu=$ gravitational constant, $\mathrm{ft}^{3} / \mathrm{s}^{2}$

$\rho=$ atmospheric density, slugs $/ \mathrm{ft}^{3}$

$\sigma \quad=$ bank angle, rad

$\phi \quad=$ latitude, rad

$\psi \quad=$ heading angle, with 0 as due east, rad

$\omega=$ natural frequency of desired drag error dynamics

$\omega_{p}=$ angular rate of planet rotation, $\mathrm{rad} / \mathrm{s}$

\section{Introduction}

Q ECOND-GENERATION reusable launch vehicles (RLVs) are $\checkmark$ needed to provide a more cost-effective and capable replacement for the U.S. space shuttle. To achieve this goal, one required area of technology development is flight mechanics. In this paper, we present and evaluate an entry guidance algorithm called evolved acceleration guidance logic for entry (EAGLE) with potential to reduce the amount of premission design effort, enlarge the window of entry opportunities, handle abort as well as nominal missions, and contribute to achieving aircraft-like operations.

Many approaches ${ }^{1-13}$ to entry guidance have been considered. See Wingrove ${ }^{1}$ for an overview and Bryson et al. ${ }^{2}$ for lessons learned from flight experience. Some entry guidance approaches involve tracking a predetermined reference trajectory; others use near realtime prediction to generate the trajectory and required controls in flight. The approach of planning and tracking aerodynamic acceleration profiles, referred to here as acceleration guidance, was developed and proven effective in the Apollo and shuttle programs. The entry guidance ${ }^{3}$ for the U.S. Space Shuttle Orbiters controls downrange by issuing bank angle magnitude commands to track a drag acceleration profile; the drag acceleration profile is also replanned onboard during entry to correct for tracking errors and null the predicted downrange error. The shuttle entry guidance controls cross range by issuing bank reversal commands to keep the heading error within certain bounds.

Entry guidance approaches for very low lift to drag ratio $(L / D)$ vehicles $(L / D<0.3)$ have been investigated for the Kistler orbital vehicle (see Ref. 4) and for Mars landing vehicles. ${ }^{5-8}$ Predictive approaches assume simple bank angle profiles, often constant magnitude with one switch in sign (bank reversal). The drag acceleration 
profile is not planned directly because it is highly constrained due to the limited lifting capability; however, it is the primary reference variable that is tracked in most of the approaches because it is closely related to measured accelerations and related to trajectory arc length by exact kinematics. Carman et al. ${ }^{7}$ uses prediction equations that are linearized about a nominal trajectory. Given the narrow envelope of flyable entry trajectories with very low $L / D$, this approach has proven viable.

For medium to high $L / D$ vehicles, there is enough flexibility to plan aerodynamic accelerations directly, knowing that there is sufficient lift to fly them, and, indeed, drag planning is the approach taken for the shuttle entry guidance. Several authors ${ }^{5,9-11}$ have proposed and evaluated variations to the shuttle entry guidance. All of these retained the basic assumption of the shuttle drag planning, namely, that the entry will evolve along a great circle arc. Lu and Hanson, ${ }^{11}$ however, included a final pre-terminal area energy management (pre-TAEM) phase in which a ground track with curvature in the lateral dimension is permitted, the purpose of this phase being to increase the capability of meeting the TAEM conditions. In developing entry guidance for the Hermes vehicle, however, Jouhaud ${ }^{12}$ determined that the shuttle entry guidance was unable to meet the required delivery accuracy, due to the decoupling of the longitudinal and lateral guidance logics and the need to make large crossrange entries near the limits of the vehicle's cross-range capability. Jouhaud proposed instead the approach of planning simultaneously drag and lateral acceleration profiles premission and tracking these profiles during the entry flight; his simulation testing indicated that this approach could meet the accuracy requirements. The dependence of the approach on a preplanned reference, however, limits its autonomy and adaptivity.

EAGLE is a direct extension of the longitudinal acceleration guidance used for the shuttle to include the lateral dimension. Whereas the shuttle drag planning is based on the assumption that the entry trajectory is a great circle arc, the trajectory planning in EAGLE accounts for trajectory curvature in the horizontal direction. With this extension, EAGLE is designed to guide large cross-range entries, as well as the descent portion of aborts that require significant cross range. Like the shuttle entry guidance, EAGLE delivers a lifting entry vehicle to a target point at which TAEM guidance takes over.

EAGLE is composed of a planning function that generates a reference trajectory and a tracking function that issues bank angle and angle-of-attack commands to follow the reference trajectory. In addition, there is higher-level logic to adjust the reference angleof-attack profile, the TAEM target point, and the tracking strategy. The planner ${ }^{14}$ uses a successive approximation approach to design reference drag and heading angle profiles. The tracking law builds on the feedback linearization-based drag tracking law introduced by Mease and Kremer ${ }^{15}$ and used for Mars landing guidance. ${ }^{5}$ In EAGLE, the bank angle command meets a weighted combination of demands for drag and heading angle tracking. Much the same as in the shuttle entry guidance, ${ }^{3}$ the angle of attack is also commanded by EAGLE as a secondary means of trajectory control, based on a washout filter that reduces high-frequency drag errors.

Other entry guidance approaches for RLVs are being developed by Dukeman, ${ }^{16}$ Zimmerman et al., ${ }^{17}$ and Shen and Lu. ${ }^{18}$ Both approaches combine planning and tracking, and the planners take into account trajectory curvature in the horizontal dimension. The planner for the first approach ${ }^{16,17}$ is based on integration of the complete three-degree-of-freedom (3-DOF) translational equations to determine an appropriate constant bank angle with one reversal. An initial temperature control phase is used to satisfy the heating rate constraint. In the second approach, ${ }^{18}$ a bank angle profile is designed under the assumption of equilibrium glide to reduce the number of equations of motion and avoid phugoid-type behavior in the planning process. Path constraints, such as the maximum heating rate, are translated to bank angle constraints. The authors note ${ }^{18}$ that the equilibrium glide assumption is not always valid; this is also indicated by the fact that the shuttle drag planning ${ }^{3}$ uses several phases, only one of which is equilibrium glide. Shen and $\mathrm{Lu}^{18}$ use a different approach in the latest part of entry when the equilibrium glide con- dition does not hold. The EAGLE planner ${ }^{14}$ requires the integration of only three kinematic equations of motion by treating the drag and lateral accelerations as the control variables in planning, and path constraints are imposed as drag constraints. This approach applies to the entire entry up to TAEM. It also suppresses phugoidlike behavior in the planning.

This paper presents the design and performance assessment of EAGLE. The performance assessment uses the simulation environment, performance criteria and scoring system provided for the advanced guidance and control (AGC) study led by NASA Marshall Space Flight Center. ${ }^{19}$ The simulation environment is the Marshall Aerospace Vehicle Representation in C (MAVERIC). ${ }^{20}$ This simulation was developed for the $\mathrm{X}-33$ program. The vehicle data represent the suborbital X-33 with the specific impulse of the engines doubled to allow the vehicle to achieve orbit. Nominal cases are defined for entry guidance testing. In this paper, we consider the return-fromorbit cases; the remaining cases are suborbital and, for the AGC study, are interpreted as abort cases. For each nominal return-fromorbit case, there are an additional 100 dispersion cases that include perturbations to initial entry conditions, air density, aerodynamic and mass properties, and navigation data, as well as actuator degradation. The scoring system quantifies the degree of success with which a guidance algorithm meets the target conditions and satisfies vehicle constraints. Other entry guidance approaches being investigated under the AGC study have been presented elsewhere. ${ }^{16-18}$ Preliminary performance results for all of the approaches were presented by Hanson and Jones, ${ }^{19}$ but do not include the EAGLE results presented in this paper.

\section{Entry Guidance Problem}

This section presents the formulation of the entry guidance problem used in the design of EAGLE. The dynamic equations, aerodynamic data, density model with altitude, etc., presented here are used exclusively in EAGLE and are, in general, simplifications of the detailed models used in high-fidelity simulation environments such as MAVERIC.

\section{Entry Dynamics}

The dynamics of atmospheric entry are expressed as a set of translational equations of motion defined in a planet-fixed coordinate frame. Because the vehicle during entry is unpowered, the total energy of the vehicle

$$
E=V^{2} / 2-\mu / r
$$

monotonically decreases along the trajectory. Energy is an appropriate independent variable for the entry dynamics. ${ }^{10,14,21}$ With energy as the independent variable, the vehicle's translational motion can be modeled by five state equations. When winds and the centripetal acceleration due to planet rotation are neglected, the equations of motion, consistent with those given in Ref. 22, except for the transformation to energy as the independent variable, are

$$
\begin{gathered}
\theta^{\prime}=-\frac{\cos \gamma \cos \psi}{r \cos \phi}\left(\frac{1}{D}\right) \\
\phi^{\prime}=-\frac{\cos \gamma \sin \psi}{r}\left(\frac{1}{D}\right) \\
r^{\prime}=-\sin \gamma\left(\frac{1}{D}\right) \\
\psi^{\prime}=\frac{\cos \psi \tan \phi \cos \gamma}{r}\left(\frac{1}{D}\right)+\frac{1}{V^{2} \cos \gamma}\left(\frac{L \sin \sigma}{D}\right)+\mathcal{C}_{\psi} \\
\gamma^{\prime}=\left(g-\frac{V^{2}}{r}\right) \frac{\cos \gamma}{V^{2}}\left(\frac{1}{D}\right)-\frac{1}{V^{2}}\left(\frac{L}{D} \cos \sigma\right)+\mathcal{C}_{\gamma}
\end{gathered}
$$


The bank angle $\sigma$ is defined such that a bank to the right is positive and zero bank corresponds to the lift vector directed upward in the longitudinal plane. The lift and drag accelerations are given by

$$
\begin{aligned}
& L=\frac{1}{2} \rho(r) V^{2} \cdot(A / m) \cdot C_{L}(\alpha, M) \\
& D=\frac{1}{2} \rho(r) V^{2} \cdot(A / m) \cdot C_{D}(\alpha, M)
\end{aligned}
$$

The density variation with altitude is modeled with the following exponential equation:

$$
\rho(r)=\rho_{0} \exp \left[-\left(r-r_{0}\right) / h_{s}\right]
$$

where the equatorial radius of the planet $r_{0}$ and the scale height $h_{s}$ and $\rho_{0}=\rho\left(r_{0}\right)$ are constants. The Coriolis terms $\mathcal{C}_{\psi}$ and $\mathcal{C}_{\gamma}$ due to planet rotation are given as

$$
\begin{gathered}
\mathcal{C}_{\psi}=-\left[2 \omega_{p} /(V D)\right](\tan \gamma \sin \psi \cos \phi-\sin \phi) \\
\mathcal{C}_{\gamma}=-\left[2 \omega_{p} /(V D)\right] \cos \psi \cos \phi
\end{gathered}
$$

A coordinate frame redefinition is used to help clarify the entry guidance problem. The usual coordinate frame, referred to as the equatorial frame, with two axes in the equatorial plane and the other toward the north pole, is rotated such that the plane given by $\phi=0$ contains both the initial and final entry positions and $\dot{\theta}>0$. This rotated frame, referred to as the guidance frame, allows us to interpret $\theta$ as the downrange angle and $\phi$ as the cross-range angle at the expense of complicating the Coriolis terms. In the next section, the planner is formulated in the guidance frame and the tracker is formulated in the equatorial frame. We will remind the reader of this, but we will use the same notation for both frames.

\section{Path and Control Constraints}

The vehicle has upper limits for dynamic pressure, aerodynamic acceleration, and heating rate. The limit on dynamic pressure is given by

$$
\bar{q}=\frac{1}{2} \rho V^{2} \leq \bar{q}_{\max }
$$

The constraint on aerodynamic acceleration is expressed as the following constraint on normal acceleration

$$
a_{n}=\sqrt{(L \cos \alpha)^{2}+(D \sin \alpha)^{2}} \leq a_{\max }
$$

The heating rate must also not exceed the design limits of the vehicle's thermal protection. Thus, the heating rate is constrained according to the heating model (based on a $1-\mathrm{ft}$ nose radius)

$$
\dot{q}=c \rho^{\frac{1}{2}} V^{3.15} \leq \dot{q}_{\max }
$$

where $c$ is a constant.

For the results presented in this paper, the following limits are imposed. The normal acceleration limit is $3.0 \mathrm{~g}$. The desired peak heat rate is $75 \mathrm{Btu} /\left(\mathrm{ft}^{2} \cdot \mathrm{s}\right)$ for the first three nominal entry cases and $60 \mathrm{Btu} /\left(\mathrm{ft}^{2} \cdot \mathrm{s}\right)$ for the remaining six nominal cases. The hard limits are taken to be $5 \mathrm{Btu} /\left(\mathrm{ft}^{2} \cdot \mathrm{s}\right)$ above the desired values. The maximum dynamic pressure is expressed in terms of a maximum $\bar{q}-\alpha$ (Ref. 23 ) of $7000 \mathrm{psf} \cdot \mathrm{deg}$ for all nine cases. For simplicity, a single nominal value of $\alpha$ is then used to convert this limit to $\bar{q}_{\max }=300 \mathrm{psf}$. For all of the results presented in this paper, no $\bar{q}-\alpha$ violations occurred. We note that the basic planner operates on a fixed $\alpha$ profile and that this profile could be used to convert the $\bar{q}-\alpha$ constraint to a drag constraint. The $\alpha$ profile adjustments discussed later are done by higher-level logic that operates outside of the planner.

From the guidance perspective, $\alpha$ and $\sigma$ are treated as the controls. For the simulation testing reported in this paper, each control is restricted to be within a certain range of values and limited by rate and acceleration constraints. The value of $\alpha$ is constrained relative to a given nominal angle-of-attack profile. The nominal value of $\alpha$ is typically large in the early part of entry to satisfy the heating constraints and eventually decreases to a lower value near that for maximum $L / D$ by the end. A limit is also placed on $\sigma$ to prevent the vehicle from reaching or exceeding a 90-deg bank angle. EAGLE is not restricted to these specific types of limits on $\alpha$ and $\sigma$.

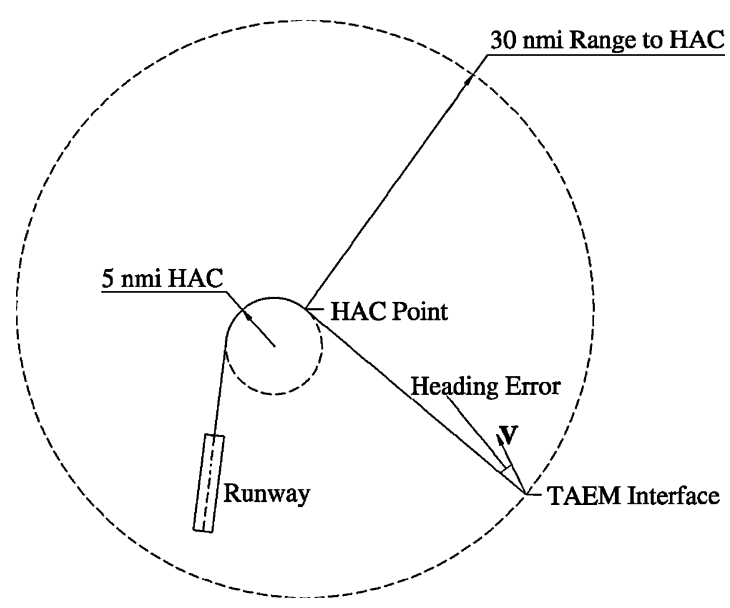

Fig. 1 Heading alignment maneuver during TAEM phase.

\section{Entry Guidance Target Conditions}

The entry guidance target is determined by the desired TAEM initial conditions. The TAEM target point marks the beginning of the TAEM phase in which the vehicle flies to a point on a heading alignment cone (HAC) and performs a turn along a 5-n mile circle to align itself with the runway ${ }^{3}$ (Fig. 1). For the performance assessment described, we adopt the TAEM point conditions as defined for the AGC study. ${ }^{19}$ The TAEM interface point is defined by a final velocity. It is desired that the TAEM interface point lie on a 30-n mile radius circle away from the HAC point (Fig. 1). At the TAEM point, the final altitude is specified and heading error to HAC should be zero. The heading error to HAC is defined as the difference between the heading of the vehicle and the required heading to be directed to the HAC point. Hanson and Jones ${ }^{19}$ provide error tolerances for the TAEM point conditions, which are also presented in the Performance Assessment section of this paper.

\section{EAGLE}

EAGLE has evolved from the acceleration entry guidance approaches developed and used in the Apollo and shuttle programs. The acceleration guidance concept is to plan and track aerodynamic accelerations, the rationale being that these variables can be related accurately to the target conditions via kinematics and measured accurately with inertial sensors. EAGLE is composed of a planning function and a tracking function that are described in the following subsections.

\section{Planning Function}

The purpose of the planning function is to determine an entry trajectory that satisfies the path constraints and target conditions. The planning function is initialized with energy-dependent reference angle-of-attack and radius profiles. The equations of motion (2) are simplified by the use of the reference radius profile to approximate $r$ and the assumption that $\cos \gamma=1$ when the right-hand sides are evaluated. The function corrects the reference radius profile to be consistent with the planned trajectory. The planning function was derived by decomposition of the entry trajectory planning problem into two subproblems, a trajectory length subproblem and a trajectory curvature subproblem, and by the use of a successive approximation approach. The state variables and equations of motion used to formulate the planner correspond to the guidance frame. A complete account of the entry planner can be found in Ref. 14; the following is a summary.

\section{Trajectory Length Subproblem}

The trajectory length subproblem is to determine a drag profile that satisfies the given path constraints and that is consistent with the required trajectory length. In the space shuttle entry guidance, ${ }^{3}$ the trajectory length subproblem is solved, under the assumption that the required trajectory length is the great circle arc length from the starting latitude, longitude point, at the altitude of the TAEM target 
point. The drag profile is divided into segments that are quadratic with respect to velocity. This allows for an analytic solution to the trajectory length equation

$$
S=-\int(V / D) \mathrm{d} V
$$

By the use of the reference $\alpha$ and $r$ profiles, the path constraints on dynamic pressure, acceleration, and heating rate [Eqs. (6-8)] are expressed as constraints on drag and determine the upper boundary of the flight corridor of allowable drag values. ${ }^{3}$

The EAGLE planning function solves the trajectory length subproblem in a similar fashion. The trajectory length is initialized as the great circle arc length to the TAEM point. Treating energy as the independent variable, the trajectory length equation becomes

$$
S=-\int(1 / D) \mathrm{d} E
$$

The current EAGLE prototype uses a three segment linear spline reference drag profile (linear in energy) with additional constraint arcs, if needed, to satisfy the path constraints. Because both drag and energy are functions of radius and velocity, the reference drag profile is used to update the radius and velocity profiles. Equation (1) for energy and Eq. (3) for drag are simultaneously solved to obtain the corresponding radius and velocity profiles by the use of the reference angle-of-attack profile and the exponential model of density variation [Eq. (4)].

With a curved trajectory, the required trajectory length $S$ is longer than the downrange given by

$$
R=-\int \frac{\cos \psi}{D \cos \phi} \mathrm{d} E
$$

To correct the value of $S$, a corresponding solution to the trajectory curvature subproblem must be determined; in the process, the bank reversal location is determined.

\section{Trajectory Curvature Subproblem}

The trajectory curvature subproblem is to determine a lateral acceleration profile that is consistent with the drag profile given by the trajectory length subproblem and that meets the desired final cross range. The lateral acceleration consistent with a given drag profile can be determined by differentiation of the drag, as given in Eq. (3) interpreted for the guidance frame, twice to obtain

$$
(L / D) \cos \sigma=(1 / b)\left(D^{\prime \prime}-a\right)
$$

where

$$
\begin{aligned}
a= & D\left(\frac{C_{D}^{\prime \prime}}{C_{D}}-\frac{C_{D}^{\prime 2}}{C_{D}^{2}}\right)+D^{\prime}\left(\frac{C_{D}^{\prime}}{C_{D}}+\frac{2}{V^{2}}\right) \\
& -\frac{4 D}{V^{4}}+\frac{1}{D V^{2}}\left(\frac{1}{h_{s}}+\frac{2 g}{V^{2}}\right)\left(g-\frac{V^{2}}{r}\right)+\left(\frac{1}{h_{s}}+\frac{2 g}{V^{2}}\right) \mathcal{C}_{\gamma}
\end{aligned}
$$

$b=-\frac{1}{V^{2}}\left(\frac{1}{h_{s}}+\frac{2 g}{V^{2}}\right)$

By the use of the reference angle of attack profile (which determines variation of $L / D$ with energy), a reference bank angle profile is constructed as

$$
\sigma=\cos ^{-1}\left[(D / b L)\left(D^{\prime \prime}-a\right)\right]
$$

The inverse cosine in Eq. (13) maps its argument into a bank angle in the range $0 \leq \sigma<\pi$. By interpretation of this bank angle value to be the magnitude of $\sigma$, it follows that only the magnitude of the lateral acceleration $|L \sin \sigma|$ is determined by the drag profile. The bank direction (bank angle sign) remains free for changing the heading and cross-range profiles. The bank direction is determined in the planning function by integration of the differential equations for heading and cross range $\left[\psi^{\prime}\right.$ and $\phi^{\prime}$ in Eqs. (2)] and by the performance of a search for the bank reversal that minimizes the final cross-range error. The point at which the reversal occurs determines the amount of time that the vehicle spends banked in the right $(+)$ and left $(-)$ directions and, thereby, determines the trajectory curvature. The energy value at which bank reversal occurs is the parameter that is adjusted by the planning function to determine the trajectory curvature and satisfy the constraint on the final cross range. The bank rate limit is respected in the computation of the bank reversal maneuver because the bank reversal is modeled as a finite transition at the maximum bank angle rate.

\section{Successive Approximation Procedure}

Because the intent of the EAGLE planning function is to accommodate entries and aborts with significant cross range, the assumption that the downrange, that is, the great circle distance, to the target is a sufficiently accurate estimate of trajectory length is not appropriate. However, with the latitude and heading angle profiles, it is possible to compute the downrange by the integration of Eq. (10). The following iterative procedure is used to improve successively the trajectory length and curvature estimates. Let $R_{d}$ denote the great circle arc distance between the initial point for the current planning step and the target point (the desired downrange). First, choose an initial estimate of the trajectory length $S_{1}$ and solve the trajectory length subproblem to obtain an initial drag profile. Second, by the use of the current estimate of the drag profile, solve the trajectory curvature subproblem. Third, based on the solution to the trajectory curvature subproblem, adjust the trajectory length by $S_{i+1}=S_{i}+\left(R_{d}-R_{i}\right)$, where $S_{i}$ and $R_{i}$ are the values from the $i$ th pass through the procedure, and solve the trajectory length subproblem to obtain a revised drag profile. Fourth, if the target error, calculated in the second step, is sufficiently small, stop; otherwise, repeat the second, third, and fourth steps.

This procedure generates reference drag and heading profiles and a reference bank command on which the tracking law operates. To compensate for tracking errors, the reference trajectory is updated at various points along the trajectory. At each update, the reference trajectory is replanned from the vehicle's current position by the use of the same successive approximation procedure, except that the estimated parameters are initialized from the preceding planned trajectory.

\section{Tracking Function}

The reference trajectory variables generated by the planning function are specified as functions of normalized energy $\tilde{E}=\left(E-E_{i}\right) /\left(E_{f}-E_{i}\right)$, where $E_{i}$ and $E_{f}$ are the initial and the desired final values. With this definition, $\tilde{E}=0$ at the start of the entry phase and reaches a value of one at the nominal TAEM condition. The reference trajectory variables are provided at a fixed number of $\tilde{E}$ points between 0 and 1 ; linear interpolation is used to obtain the reference variables at intermediate values of $\tilde{E}$.

The bank angle command is derived by initially treating $u_{D}=(L / D) \cos \sigma$ and $u_{\psi}=(L / D) \sin \sigma$ as two independent inputs and assuming that $\alpha(E)=\alpha_{\text {ref }}(E)$. Considering drag and heading as outputs of the system dynamics and $u_{D}$ and $u_{\psi}$ as inputs, the inputs and outputs are related via the equations

$$
\begin{gathered}
D^{\prime \prime}=a+b u_{D} \\
\psi^{\prime}=\frac{\cos \gamma \cos \psi \tan \phi}{r D}+\frac{1}{V^{2} \cos \gamma} u_{\psi}+\mathcal{C}_{\psi}
\end{gathered}
$$

where $a$ and $b$ are as defined in Eqs. (12), except that the tracking law is formulated in the equatorial plane. Thus, $u_{D}$ and $u_{\psi}$ can be used to control the drag dynamics, and the heading dynamics, respectively. We design $u_{D}$ so that, in the absence of a modeling error, drag tracks the reference drag profile according to specified second-order linear error dynamics. Letting $D_{\text {ref }}$ represent the reference drag and $D_{\text {ref }}^{\prime}$ and $D_{\text {ref }}^{\prime \prime}$ represent the derivatives of the reference drag with respect 
to energy, the specified second-order linear error dynamics are

$$
\begin{aligned}
& \left(D_{\text {ref }}^{\prime \prime}-D^{\prime \prime}\right)+2 \zeta \omega(\bar{q})\left(D_{\text {ref }}^{\prime}-D^{\prime}\right)+\omega^{2}(\bar{q})\left(D_{\text {ref }}-D\right) \\
& \quad+k_{1} \int\left(D_{\text {ref }}-D\right) \mathrm{d} E=0
\end{aligned}
$$

The parameters $\omega(\bar{q})$ and $\zeta$ determine the proportional and the derivative gains for the drag tracking law. Through substitution for $D^{\prime \prime}$ from Eq. (14), the input $u_{D}$ required to track $D_{\text {ref }}$ is given by

$$
\begin{aligned}
u_{D}= & \frac{1}{b}\left[-a+D_{\mathrm{ref}}^{\prime \prime}+2 \zeta \omega(\bar{q})\left(D_{\mathrm{ref}}^{\prime}-D^{\prime}\right)+\omega^{2}(\bar{q})\left(D_{\mathrm{ref}}-D\right)\right. \\
& \left.+k_{1} \int\left(D_{\mathrm{ref}}-D\right) \mathrm{d} E\right]
\end{aligned}
$$

Similarly, we design $u_{\psi}$ so that the heading angle tracks the reference heading profile according to the first-order error dynamics

$$
\left(\psi_{\mathrm{ref}}^{\prime}-\psi^{\prime}\right)+k_{2} \omega(\bar{q})\left(\psi_{\mathrm{ref}}-\psi\right)+k_{3} \int\left(\psi_{\mathrm{ref}}-\psi\right) \mathrm{d} E=0
$$

where $\psi_{\text {ref }}$ represents the reference heading and $\psi_{\text {ref }}^{\prime}$ represents the first derivative of heading with respect to energy. By substitution for $\psi^{\prime}$ from Eqs. (2), we get

$$
\begin{aligned}
u_{\psi}= & V^{2} \cos \gamma\left[-\frac{\cos \gamma \cos \psi \tan \phi}{r D}-\mathcal{C}_{\psi}+\psi_{\mathrm{ref}}^{\prime}\right. \\
& \left.+k_{2} \omega(\bar{q})\left(\psi_{\mathrm{ref}}-\psi\right)+k_{3} \int\left(\psi_{\mathrm{ref}}-\psi\right) \mathrm{d} E\right]
\end{aligned}
$$

The natural frequency is computed by the use of

$$
\omega(\bar{q})=\left[2\left(\bar{q} / \bar{q}_{\max }\right)-\left(\bar{q} / \bar{q}_{\max }\right)^{2}\right] \omega_{0}
$$

When the natural frequency is scheduled in this manner, the natural frequency is small in the initial part of the trajectory where the dynamic pressure is low. This prevents the bank angle from saturating in response to small drag errors in low dynamic pressure conditions. Because the maximum bank rate may not be too large (about $5 \mathrm{deg} / \mathrm{s}$ for the results presented in this paper), the prevention of bank angle saturation makes it more likely that the appropriate bank angles can be achieved as the dynamic pressure and drag increase.

For the results presented in this paper, we use the following values for the parameters in the tracking laws. Our specification of the natural frequency, damping ratio, and integral gain in the second-order error dynamics, Eq. (15), is indirect. With time as the independent variable, there is a corresponding linear second-order equation, and we specify the corresponding parameters with a value of 0.237 for $\omega_{0}$ and a value of 0.45 for $\zeta$. The transformations given in Ref. 5 provide the equivalent parameters for the energy domain. The value used for $k_{1}$ is $1.05 \times 10^{-5}$, which gives the integral term a minor influence in the tracking law. Values of 0.18 and 0 are used for $k_{2}$ and $k_{3}$, respectively. The value of $\bar{q}_{\max }$ is $500 \mathrm{psf}$.

We present two ways of allocating the drag and heading demands, $u_{D}$ and $u_{\psi}$, to the guidance command variables $\sigma$ and $\alpha$. In the first, both $\sigma$ and $\alpha$ are treated as primary control variables; in the second, $\sigma$ is the primary control variable and $\alpha$ is a secondary control variable.

With both $\sigma$ and $\alpha$ as primary control variables, there may exist values of $(\sigma, \alpha)$ such that both $u_{D}$ and $u_{\psi}$ are achieved exactly. The angle of attack is then selected such that $L / D=\sqrt{ }\left(u_{D}^{2}+u_{\psi}^{2}\right)$; the bank angle is given by $\sigma=\tan ^{-1}\left(u_{\psi} / u_{D}\right)$. Given the constraints on $\sigma$ and $\alpha$, it is not always possible to achieve $u_{D}$ and $u_{\psi}$ exactly, and some logic for compromise is required. The problem with this approach is that the direct effect of changing $\alpha$ from $\alpha_{\text {ref }}$ on the drag acceleration has not been accounted for in the formulation.

For the results presented in this paper, the following allocation approach is used. The bank angle is treated as the primary control variable; the angle of attack is treated as a secondary control variable. The bank angle is selected as a compromise between meeting the drag and the heading demands $u_{D}$ and $u_{\psi}$. Different compromises are made in different portions of the entry. The bank angles corresponding to $u_{D}$ and $u_{\psi}$ are first computed by the use of measured values of $L$ and $D$ and the definitions

$$
\begin{gathered}
\left|\sigma_{D}\right|=\cos ^{-1}\left(D u_{D} / L\right) \\
\sigma_{\psi}=\sin ^{-1}\left(D u_{\psi} / L\right)
\end{gathered}
$$

The drag tracking demand only dictates the magnitude of bank angle. The sign of $\sigma_{D}$ is set equal to the sign of the reference bank angle $\left(\sigma_{\text {ref }}\right)$ generated by the planning function.

Except during the bank reversals, the commanded bank angle $\sigma_{\mathrm{cmd}}$ is calculated as the following weighted average of $\sigma_{D}$ and $\sigma_{\psi}$ :

$$
\sigma_{\mathrm{cmd}}=w(E) \operatorname{sgn}\left(\sigma_{\mathrm{ref}}\right) \sigma_{D}+[1-w(E)] \sigma_{\psi}
$$

where $w(E)$ is a continuous function of energy and takes values between zero and one. In the initial portion of the entry, $w(E)$ takes a value one and emphasizes drag tracking completely. In the latter portion $w$ is chosen in the range of 0.6-0.7. Drag tracking is emphasized initially because drag tracking is quite challenging by itself and requires full attention; also, if drag is tracked accurately, then nominally the reference heading will be followed. Moreover, at each planning update, the reference heading angle profile is designed to start from the current heading angle and, hence, any heading error is nullified. After the final bank reversal, there are no more updates and heading tracking must receive some attention; this issue is addressed further in the next section.

During a bank reversal, the bank angle command is open-loop, taking values from the same reversal profile used in the planning, namely, a linear-in-time profile with slope equal to the maximum bank angle rate. A slight modification to this strategy is described in the next section.

The angle-of-attack command nominally follows the reference profile that is used by the planning function to generate the drag and heading profiles. However, angle of attack adjustments about the reference are used to compensate for transient errors in drag tracking that bank angle modulation cannot handle, similarly to what is done in the shuttle entry guidance. ${ }^{3}$ Because the derivation of $\sigma_{D}$ did not account for deviations in $\alpha$ from $\alpha_{\text {ref }}$, the adjustment of $\alpha$ in this manner requires justification. The justification ${ }^{3}$ is that $\alpha$-modulation is responding to the high-frequency component of the drag error, whereas the $\sigma_{D}$ command is responding to the lowfrequency component of the drag error. To achieve this effect, the drag error is converted to an angle-of-attack adjustment by the use of the coefficient of drag and $\bar{q}$, and then passed through the washout filter $s /(s-p)$. The washout filter allows $\alpha$ modulation to compensate for fast drag error behavior, but not for slower behavior that can and should be compensated for by bank angle modulation. The pole $p$ determines the break frequency. In the results presented later, $p=-0.002$. Also, the angle-of-attack adjustment is limited to \pm 5 deg from the reference value and the bank angle and angle-of-attack commands are rate limited to $\pm 5 \mathrm{deg} / \mathrm{s}$ to be consistent with the vehicle model. It is expected that a higher bank rate would improve tracking, whereas a lower bank rate limit would make tracking more difficult.

\section{Additional Logic in EAGLE}

In addition to the planning and tracking functions just presented, there is higher-level logic in EAGLE to 1) manage the final bank reversal location via adjustments to the location of a major breakpoint of the reference angle of attack profile, 2) control the final heading angle via adjustments to the TAEM point, and 3) adjust the tracking strategy during and after the final bank reversal to ensure that the target conditions are met with sufficient accuracy. These features significantly improve the performance of EAGLE for the return-from-orbit test cases described in the Performance Assessment section. 


\section{Bank Reversal Management}

After the vehicle has performed its last bank reversal, the reference heading profile is not updated. If the heading tracking is inaccurate, an unacceptable cross-range error could result. Because there must be a compromise in meeting the drag and heading tracking demands, $w=0$ in Eq. (22), which would ignore the drag tracking demand is not viable. Thus, to avoid unacceptable cross-range errors, the final bank reversal should occur relatively close to the target. For short trajectories, a single bank reversal may be adequate, whether it be early or late in the entry phase. For longer cases, two potential strategies are 1) plan the first reversal close to TAEM point or 2) plan more than one bank reversal with the last reversal sufficiently near the TAEM point. Both strategies are used by the planning function, the choice depending on the cross range to be covered. It is not easy to quantify generally how near the TAEM point the last bank reversal should be located for entry trajectories with different down- and cross-range requirements. The Performance Assessment section gives an example of a good reversal location for the return-from-orbit trajectories considered there. We expect that more general bank reversal management logic would be developed for an operational system.

\section{Planning a Single Bank Reversal}

The angle-of-attack profile has a significant effect on the placement of the bank reversal by the planner. The planning function, thus, includes a feedback-based procedure to adjust the angle-ofattack profile such that the final bank reversal is close to the TAEM point. Because of the heating constraint, the angle of attack needs to be high in the initial phase, close to $50 \mathrm{deg}$ from $\tilde{E}=0$ to 0.5 for the entry cases considered in this paper. Hence, the freedom in angle of attack is between $\tilde{E}=0.5$ and 1 .

Figure 2 illustrates the effect of a change in the angle-of-attack profile on a trajectory. Cross range is plotted vs downrange in Fig. 2 for $\tilde{E}>0.5$ to illustrate the effect of an $\alpha$ profile variation. The curvature of the trajectory with the higher angle-of-attack profile is less than that with the lower angle-of-attack profile. The reference drag profiles for the two cases are almost the same. Under the assumption that the drag profile is invariant under the type of angle-of-attack profile changes we are considering, the effect of the $\alpha$-profile change on the lateral curvature can be explained. Tracking the drag profile requires a certain $(L / D) \cos (\sigma)$ profile. In the range of angle of attack we are using, increasing angle of attack decreases $L / D$; thus, a smaller value of $\sigma$ is required to achieve the necessary $(L / D) \cos (\sigma)$. This, in turn, implies that $(L / D) \sin (\sigma)$ will decrease, thereby decreasing the turn rate $\psi^{\prime}$.

A change in trajectory curvature causes the bank reversal point to shift. For test cases in which significant cross range is required,
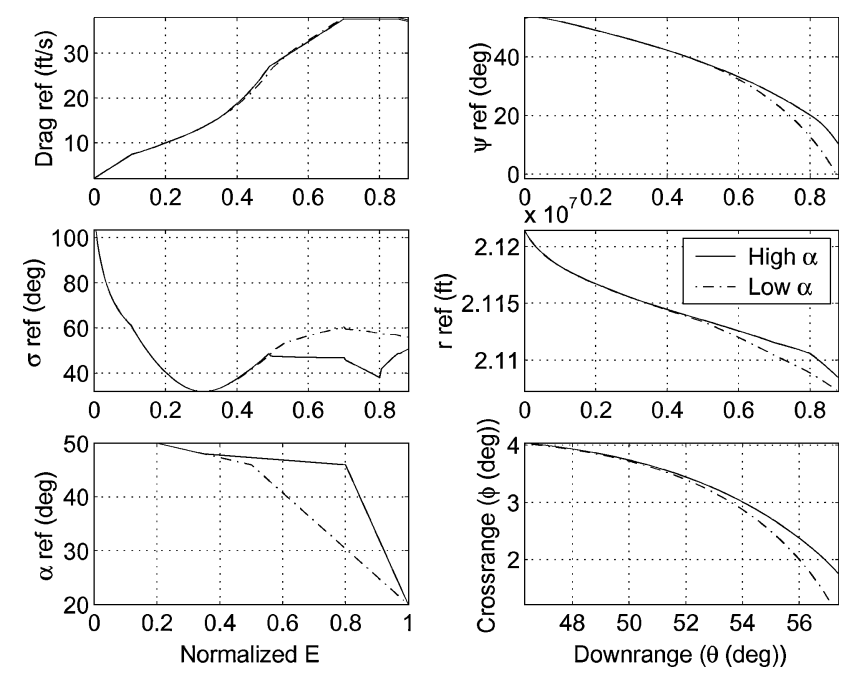

Fig. 2 Effect of shift of $\alpha$ profile breakpoint on trajectory curvature.
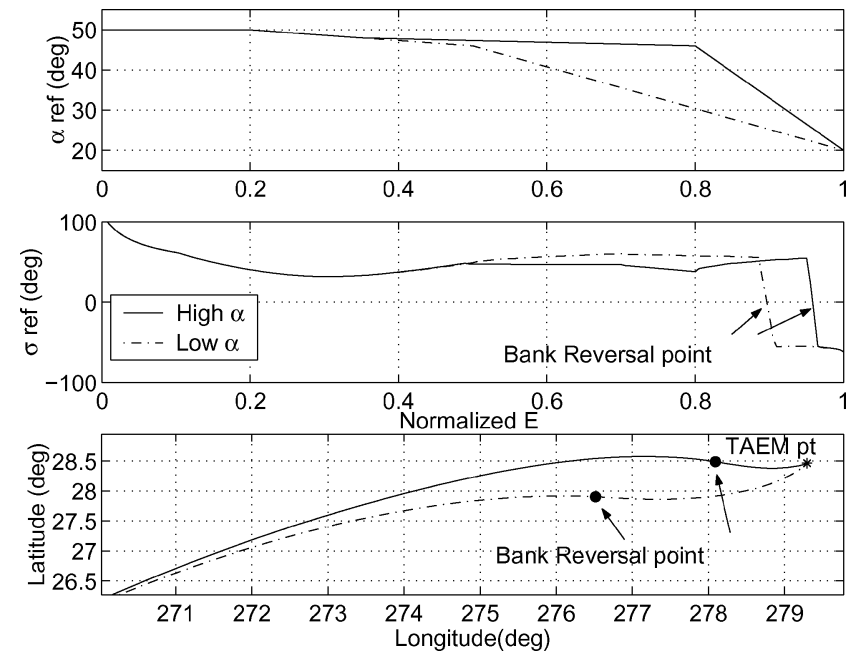

Fig. 3 Effect of shift of $\alpha$ profile breakpoint on bank reversal point.

bank reversals occur beyond $\tilde{E}=0.6$. The reference angle-of-attack profile in the planning function is represented as a continuous spline with segments that vary linearly with $\tilde{E}$. An important breakpoint in this spline is where the angle of attack begins to decrease from the initial high constant value. When the breakpoint is shifted to a higher value of $\tilde{E}$, the planning function shifts the bank reversal point to a higher value of $\tilde{E}$ to reach the TAEM point. This phenomenon is demonstrated in Fig. 3.

To place the bank reversal at $\tilde{E}_{\text {revdes }}$, the desired energy value, a proportional feedback law has been implemented in the planning function. Each time the planning function is called to update the trajectory, the energy breakpoint $\tilde{E}_{\mathrm{bp}}$ for the angle-of-attack profile is adjusted by a proportional feedback term, namely,

$$
\tilde{E}_{\mathrm{bp}}(i)=\tilde{E}_{\mathrm{bp}}(i-1)+K_{\mathrm{rev}}\left[\tilde{E}_{\text {revdes }}-\tilde{E}_{\mathrm{rev}}(i-1)\right]
$$

where $\tilde{E}_{\mathrm{bp}}(i)$ is the location of the breakpoint at the $i$ th update of the trajectory, $K_{\text {rev }}$ is the proportional feedback gain, $\tilde{E}_{\text {revdes }}$ is the desired location of bank reversal, and $\tilde{E}_{\mathrm{rev}}(i-1)$ is the planned location of bank reversal at the $(i-1)$ th update. After the angleof-attack profile is updated, the reference trajectory is recomputed based on the new angle-of-attack profile, and the new location of the bank reversal is obtained.

\section{Planning Two Bank Reversals}

With all other initial entry conditions the same, as the required cross range decreases, the value of $\tilde{E}$ at which the bank reversal takes place decreases. The angle-of-attack breakpoint variation may not shift the bank reversal location sufficiently. In such a situation, a two-reversal approach is implemented by the use of the one-reversal planner. First, the planner determines a singlereversal trajectory; however, the reversal is actually executed slightly earlier than planned. The premature execution of the bank reversal creates the need for a second, corrective bank reversal near the end of the entry phase. The same planning function, applied after the first reversal, determines the location of the second reversal.

\section{Meeting the Final Heading Constraint}

As stated earlier, there is a requirement that the vehicle is headed toward the HAC point when it reaches the TAEM interface circle. Because the planner only determines one bank reversal in a trajectory update, and does so to minimize the cross-range error, the final heading cannot be specified. Instead, the freedom to select a TAEM point on the interface circle has been used to meet the heading constraint. The TAEM point is set initially as the intersection of the interface circle and the great circle plane containing the HAC point and the initial entry point. The following method is then used to reassign the TAEM point each planning update. First the trajectory 
is planned by the use of the current TAEM point location. Then, the final velocity vector is determined from the planned trajectory. Finally, the TAEM point is relocated along the interface circle such that the velocity vector is aligned with the HAC point. This method is used before the first reversal in trajectories with high initial cross range and between the first and the second reversal for trajectories with low initial cross range. The TAEM point is relocated once every update and convergence is usually achieved after a few updates.

The law for relocating the TAEM point, used in the preceding approach, is based on the assumption that the movement of the TAEM point does not significantly affect the trajectory shape, nor the direction of the velocity vector at the end of the trajectory. The movement of the TAEM point changes the cross range, and so the planning function needs to change the location of bank reversal to null the cross-range error. If the bank reversal point is located at a large distance from the TAEM point, a small adjustment in bank reversal can nullify the cross-range error without affecting the final heading. However, when the bank reversal is located close to the TAEM point, change in cross range significantly affects the bank reversal point and the resulting final heading. As a consequence, the TAEM point iteration may not converge. To avoid this problem, the TAEM point adjustment is halved at each iteration, if the final reversal is located close to the target.

\section{Control Logic Near Last Bank Reversal}

Accurate tracking during and following the last bank reversal is very important for meeting the TAEM point constraints with sufficient accuracy. For most of the trajectory before the last reversal, the bank angle commands are dedicated to drag tracking. To initiate the bank reversal, the sign of the bank angle command for drag tracking $\sigma_{D}$ is switched. Because the bank commands pass through a software rate and acceleration limiter, this sign switch results in a ramp-shaped bank command. Because rate and acceleration limits are also implemented in the planning function, the reference bank profile also has a ramp shape at the bank reversal. For accurate tracking of the reference bank reversal maneuver, the following control logic is implemented. Close to the last reversal, if the vehicle is banked less than the reference bank angle, the bank angle command is frozen and the sign switch of $\sigma_{D}$ is slightly delayed beyond the planned reversal point. Similarly, if the vehicle is banked more than the reference bank angle, the sign of $\sigma_{D}$ is switched slightly earlier than the planned bank reversal instant. This strategy results in a closer match between the reference and actual bank angles during the reversal.

Logic is also added to prevent any updates of the trajectory slightly before or during the bank reversal. Because the reference drag profile consists of linear segments, when the trajectory is updated by the planning function, a small but discontinuous change in the reference drag takes place. This results in transients in the bank angle command, and the presence of transients near the bank reversal may result in poor tracking during the reversal.

After the last reversal, it is important to first correct the drag error caused by the reversal and then to emphasize heading tracking. During the bank reversal, as the lift vector rotates and crosses the vertical plane, the flight-path angle increases and this causes the drag to reduce. To increase drag, the drag tracking law in Eq. (21) demands a bank angle larger than the reference. Heading tracking is emphasized only after the bank angle demand for drag tracking becomes less than bank demand for heading tracking. A weighting $w \approx 0.4$ in the bank command law [see Eq. (22)] is used after the drag recovery. This logic has proven useful in meeting the TAEM conditions as demonstrated in the following section.

\section{Performance Assessment}

This section presents the performance of EAGLE in the MAVERIC simulation environment. The vehicle model used in the test cases is representative of the X-33 (Ref. 24). There are nine nominal return-from-orbit test cases, referred to by entry quidance (EG) EG13-EG21, defined for the AGC study. ${ }^{19}$ Cases EG13-EG18 represent entries from the International Space Station orbit, which has an inclination of $51.6 \mathrm{deg}$, whereas cases EG19-EG21 represent entries from low Earth orbit, which has an inclination of $28.5 \mathrm{deg}$. EG13-EG15 are late-entry cases and have shorter ranges to TAEM as compared to those of EG16-EG18, which are early-entry cases. EG13, EG16, and EG19 have low cross-range requirements, in that the initial heading is almost directly toward the HAC point. Cases EG14, EG17, and EG20 are high cross-range variants of EG13, EG16, and EG19, respectively, with initial headings to the right of the HAC point. Similarly EG15, EG18, and EG21 are high crossrange variants of EG13, EG16, and EG19, with initial headings to the left of the HAC point.

In accordance with the AGC study ${ }^{19}$ rules for testing cases EG13EG21, the MAVERIC simulations presented in this paper have been run in 3DOF. The commanded bank angle and angle of attack are each delayed by about $1 \mathrm{~s}$ and subjected to magnitude, rate, and acceleration limits, to approximate the actual bank angle and angleof-attack responses, before they are inserted into the equations of motion and integrated.

Figures 4 and 5 show the ground tracks for the nominal entry cases as flown by EAGLE. A summary of the initial conditions and desired target (TAEM point) conditions is presented in

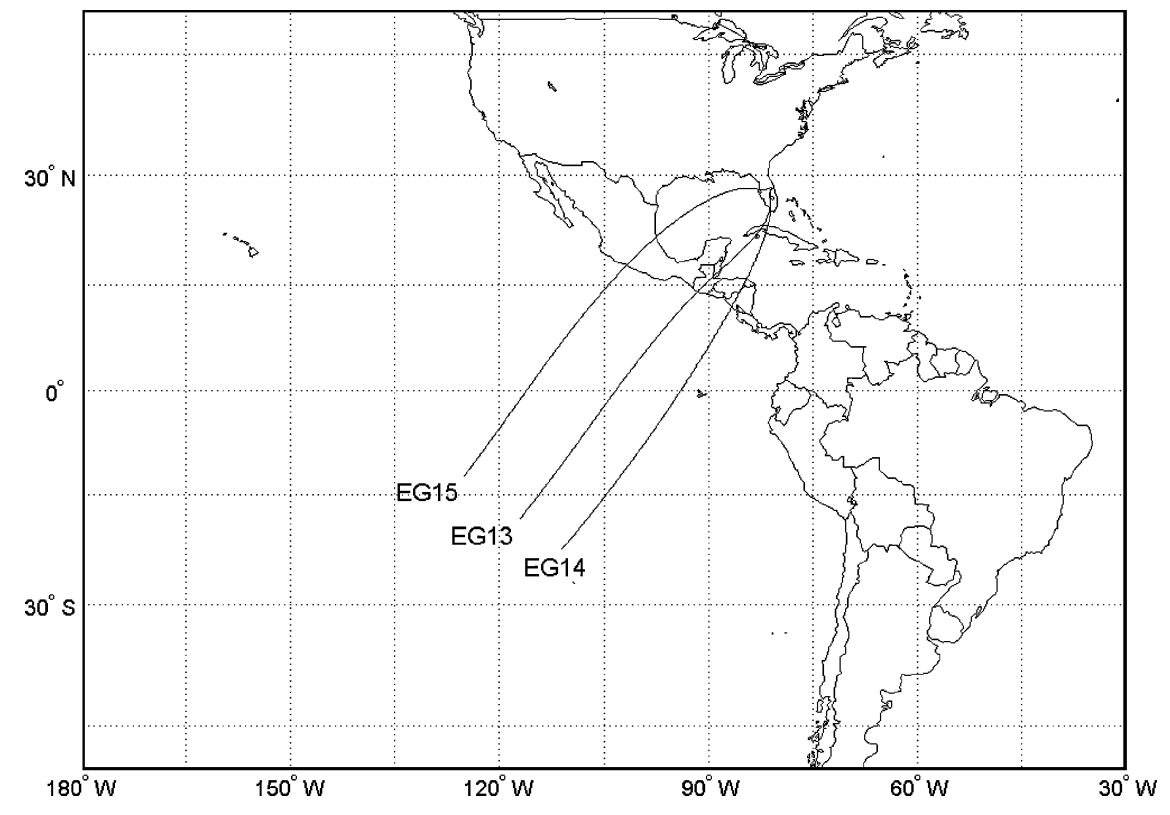

Fig. 4 Ground track for EG13-EG15. 
Table 1 Initial and target conditions

\begin{tabular}{lccccccccc}
\hline \hline Case & $h_{0}, \mathrm{ft}$ & $\phi, \mathrm{deg}$ & $\theta, \mathrm{deg}$ & $V_{0}, \mathrm{ft} / \mathrm{s}$ & $\psi_{0}, \mathrm{deg}$ & $\phi_{\text {HAC }}$ & $\theta_{\text {HAC }}$ & $h_{\text {TAEM }}$ & $V_{\text {TAEM }}$ \\
\hline EG13 & 398,681 & -18.255 & -117.01 & 25,007 & 38.329 & 28.61 & -80.496 & $99,827.3$ & $2,979.0$ \\
EG14 & 402,093 & -22.510 & -111.01 & 25,004 & 39.856 & 28.61 & -80.496 & $99,827.3$ & $2,979.0$ \\
EG15 & 394,928 & -12.223 & -125.01 & 25,009 & 36.812 & 28.61 & -80.496 & $99,827.3$ & $2,979.0$ \\
EG16 & 399,800 & -29.516 & -127.50 & 25,020 & 43.447 & 28.67 & -80.506 & $96,560.0$ & $3,008.08$ \\
EG17 & 403,885 & -33.263 & -122.50 & 25,017 & 46.06 & 28.67 & -80.506 & $96,560.0$ & $3,008.08$ \\
EG18 & 394,134 & -23.751 & -134.50 & 25,024 & 40.409 & 28.67 & -80.506 & $96,560.0$ & $3,008.08$ \\
EG19 & 399,114 & -2.3046 & -141.72 & 24,417 & 59.854 & 28.67 & -80.506 & $99,827.3$ & $3,008.08$ \\
EG20 & 400,522 & -8.446 & -137.72 & 24,416 & 61.026 & 28.67 & -80.506 & $99,827.3$ & $3,008.08$ \\
EG21 & 409,606 & 22.83 & -157.72 & 24,410 & 71.4535 & 28.67 & -80.506 & $99,827.3$ & $3,008.08$ \\
\hline \hline
\end{tabular}

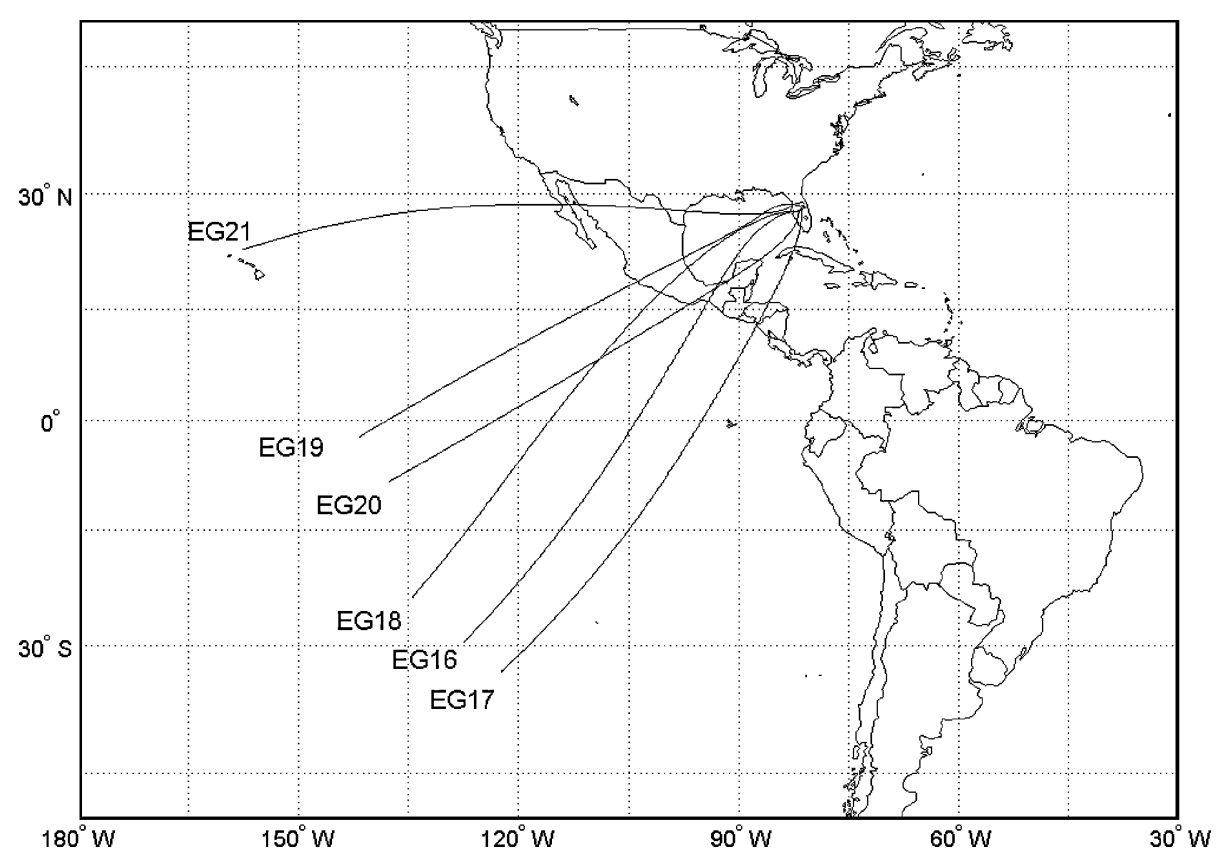

Fig. 5 Ground track for EG16-EG21.

Table 1. The initial conditions correspond to the vehicle state just after the completion of the deorbit burn. The entry phase is not started from this state because the aerodynamic forces are very small, and there is essentially no capability to alter the flight path. After the deorbit burn, the vehicle enters a transition phase during which no guidance laws are operated, and constant $\sigma$ and $\alpha$ are maintained by the flight controller. The entry phase begins and EAGLE starts operating when the aerodynamic drag rises to about $4 \mathrm{ft} / \mathrm{s}^{2}$. The initial conditions for entry are obtained by integration of the vehicle dynamics through the transition phase with constant $\sigma$ and $\alpha$. AGC study rules provide flexibility to choose the values $\sigma$ and $\alpha$ during transition. After some trial simulation runs, $\sigma=15 \mathrm{deg}$ and $\alpha=50 \mathrm{deg}$ were chosen. These values provided the best entry conditions for meeting the heating and downrange requirements.

For the AGC study, the test cases are divided into two groups: EG13-15 and EG16-21. Because the test cases are significantly different in terms of the total range covered (EG13-15 have smaller range than EG16-21), guidance designers are allowed to submit a different entry guidance algorithm for each group. The results presented here, however, are obtained with a single version of EAGLE, that is, a single set of gains and other guidance constants is used for all cases and all of the other logic is identical.

Two simulations are first presented to illustrate certain features of EAGLE in the planning and tracking of entry trajectories. Figure 6 shows some key variables for the simulated entry by the use of the EAGLE algorithm for the nominal EG16 case. The variables are plotted against normalized energy $\tilde{E}$. The planner generates reference heading and drag profiles, updating these profiles every $25 \mathrm{~s}$. The upper bound on drag, computed from the maximum limits of heat rate, normal acceleration, and dynamic pressure, is also plot-
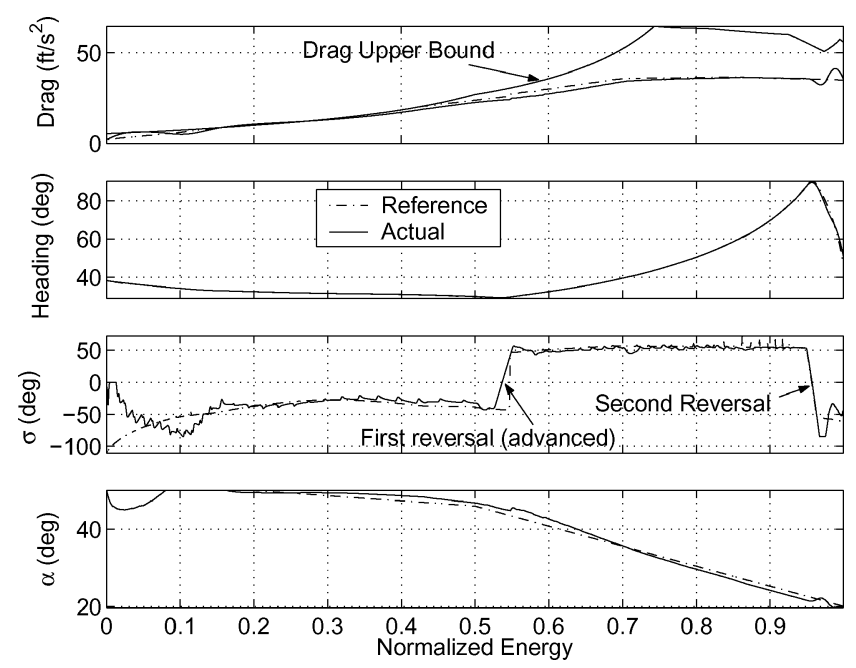

Fig. 6 Trajectory profiles for EG16.

ted in Fig. 6. The reference drag profile is constrained by the drag bound (computed from the heat rate limit) between $\tilde{E}=0.15$ and 0.45 . To ensure that the errors in drag tracking do not cause constraint violations, the drag bound is lowered by about $2.3 \mathrm{ft} / \mathrm{s}^{2}$. The planning function generates a reference bank angle profile that is consistent with the drag and heading profiles. These reference profiles are also plotted in Fig. 6. The commanded bank angle and angle of attack are shown, along with the actual simulated drag and heading angle. The heading and drag reference profiles are tracked 

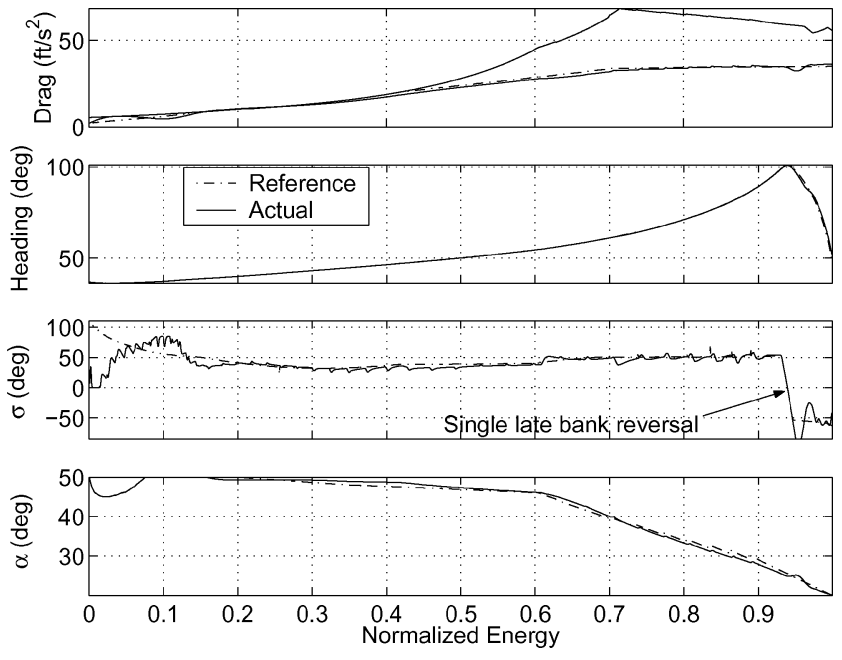

Fig. 7 Trajectory profiles for EG18.

closely. For EG16, EAGLE plans and commands two bank reversals (at the normalized energies of 0.536 and 0.956 ) because the first reversal is quite early and close to the $\tilde{E}=0.5$ breakpoint in the angle-of-attack profile. The planning function identifies the need for the second bank reversal immediately after the first trajectory is planned. As described earlier, the guidance logic advances the first reversal (by about $\tilde{E}=0.035$ ) and then plans the second reversal after the first reversal is complete. The effect of the special control logic at the last bank reversal can also be seen in this test case. The second bank reversal is accurately tracked, and the heading tracking begins only after the drag error due to the bank reversal has been reduced. The angle of attack normally follows the reference value and responds only to transient errors in drag tracking, a result of the washout filter in the feedback path.

Figure 7 shows a simulation of the nominal test case EG18, an entry trajectory that requires a large cross range. When the planning function is called initially, the bank reversal is located at about $\tilde{E}=0.8$, with the nominal angle-of-attack profile. The logic described in the preceding section shifts the angle-of-attack profile

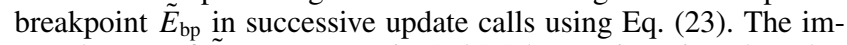
posed range of $\tilde{E}_{\mathrm{bp}}$ movement is $(0.5,0.85) . K_{\text {rev }}$ is assigned a value of 1.5. A good value for $\tilde{E}_{\text {revdes }}$ is 0.95 . However, $\tilde{E}_{\text {revdes }}$ is actually computed according to

$$
\tilde{E}_{\text {revdes }}=0.95-0.1(0.90-\tilde{E}), \tilde{E}_{\text {revdes }} \in[0.925,0.95]
$$

As $\tilde{E}$ increases, $\tilde{E}_{\text {revdes }}$ is gradually moved from 0.925 to 0.95 . This adjustment of $\tilde{E}_{\text {revdes }}$ is necessary because the planning function also adjusts the location of bank reversal point in every trajectory update to nullify tracking errors. The preceding logic ensures that $E_{\mathrm{bp}}(i)$ does not locate the reversal at 0.95 from the beginning. For the EG18 test case in Fig. 7, the bank reversal shifts from about 0.8 to finally occur at 0.939 . The reference drag and heading profiles are again tracked closely.

Figure 4, as mentioned earlier, shows the ground tracks for the nominal cases EG13-EG15 and Fig. 5 shows the ground tracks for the nominal cases EG16-EG21. In EG13, EG16, and EG19, the vehicle is headed directly toward the HAC point at entry. These cases require two bank reversals, and the effects of the bank reversals are noticeable on the ground tracks. The remaining nominal cases have significant right and left cross-range offsets, and so a single late reversal is used. All of the cases have significant lateral motion, and EAGLE uses the bank reversals and adjusts the TAEM point effectively to reach the TAEM interface circle with minimal downrange, cross-range, and heading errors.

For each nominal case EG13-EG21, there are an additional 100 dispersion cases that include perturbations to initial entry conditions, air density, vehicle aerodynamic and mass properties, and navigation data, as well as actuator degradation. Figures 8 and 9

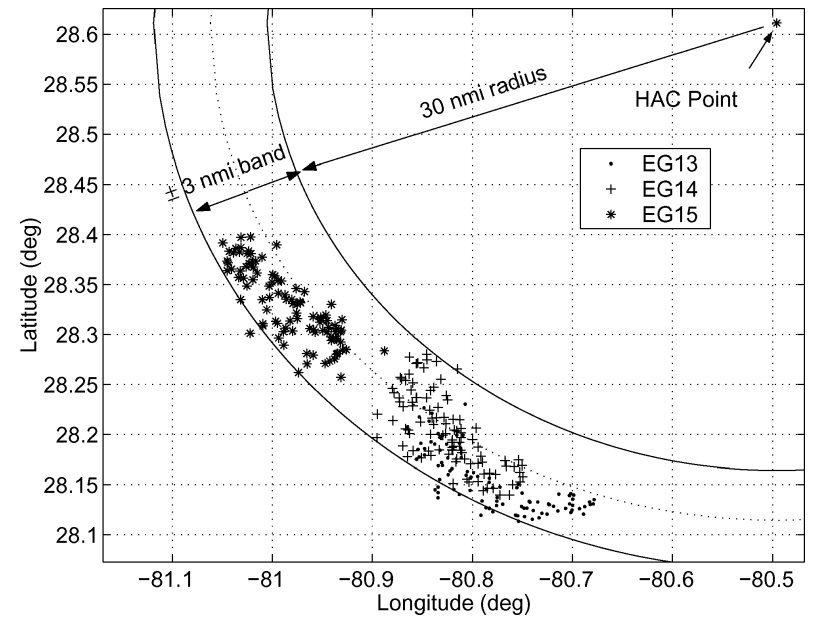

Fig. 8 TAEM point dispersions for EG13-EG15.

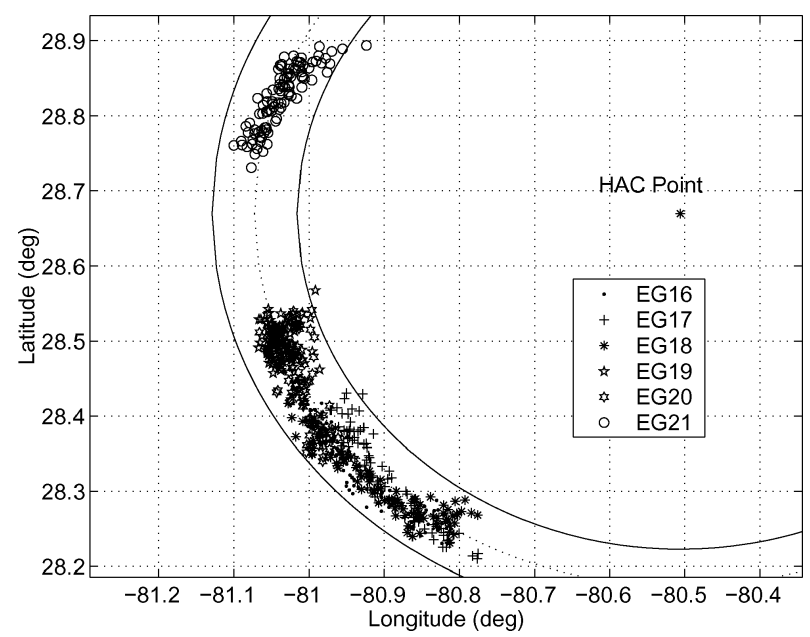

Fig. 9 TAEM point dispersions for EG16-EG21.
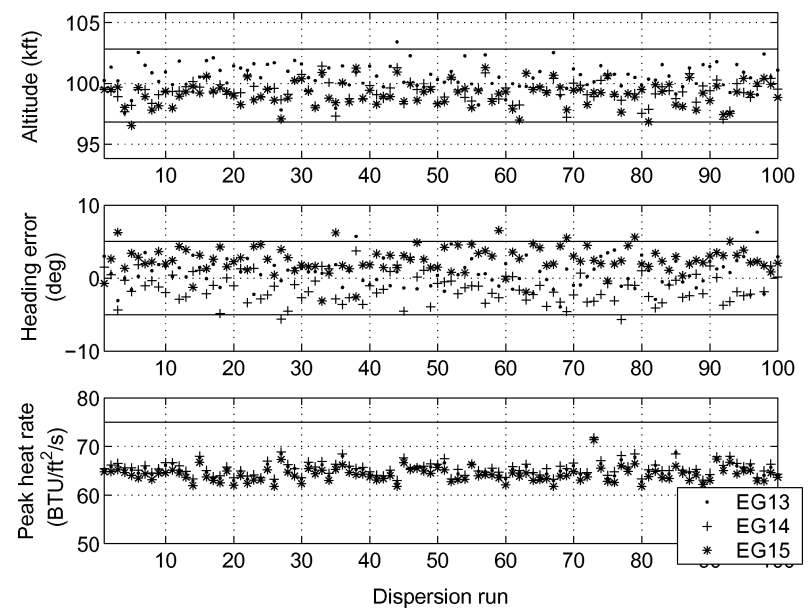

Fig. 10 Results of dispersion runs for EG13-EG15.

show closeups around the HAC point. The endpoints of the simulated trajectories are plotted for all of the dispersion cases. Each simulation is terminated when the vehicle velocity becomes equal to the TAEM velocity given in Table 1 . Figures 8 and 9 show a portion of the 30-n mile circle around the HAC point and the tolerable error band of $\pm 3 \mathrm{n}$ mile. The endpoints of almost all of the trajectories lie within the \pm 3 -n mile band.

The actual heading errors, altitude errors (relative to the TAEM altitude in Table 1) and the peak heat rates are plotted in Figs. 10 
Table 2 Flight parameters and scoring method developed by NASA MSFC ${ }^{19}$

\begin{tabular}{|c|c|c|c|c|}
\hline Flight parameter & Nominal & 0 Score & 1 Score & Weight \\
\hline Maximum negative $N_{z}, g$ & 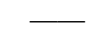 & $\geq 3.5$ & $\leq 3.0$ & 0.1 \\
\hline Maximum $\bar{q}-\alpha$ & & $\geq 8000$ & $\leq 7000$ & 0.05 \\
\hline Maximum heat rate, $\mathrm{Btu} /\left(\mathrm{ft}^{2} \cdot \mathrm{s}\right)$ & & $\geq 80$ & $\leq 75$ & 0.19 \\
\hline Heading error at TAEM, deg & 0 & $\geq 10$ & $\leq 5$ & 0.13 \\
\hline Altitude at TAEM, $10^{3} \mathrm{ft}$ & 99.827 & |error| & |error| & 0.16 \\
\hline Range to HAC at TAEM, $n$ mile & 30 & $\begin{array}{l}\geq \pm 6 \\
\mid \text { error| } \\
\geq \pm 6 \\
\geq-1,\end{array}$ & $\begin{array}{l}\leq \pm 3 \\
\mid \text { error| } \\
\leq \pm 3 \\
\geq-23\end{array}$ & 0.23 \\
\hline FPA at TAEM, deg & & $\leq-25$ & $\leq-4$ & 0.08 \\
\hline |Bank angle| at TAEM, deg & 0 & $\geq 60$ & $\leq 50$ & 0.06 \\
\hline
\end{tabular}
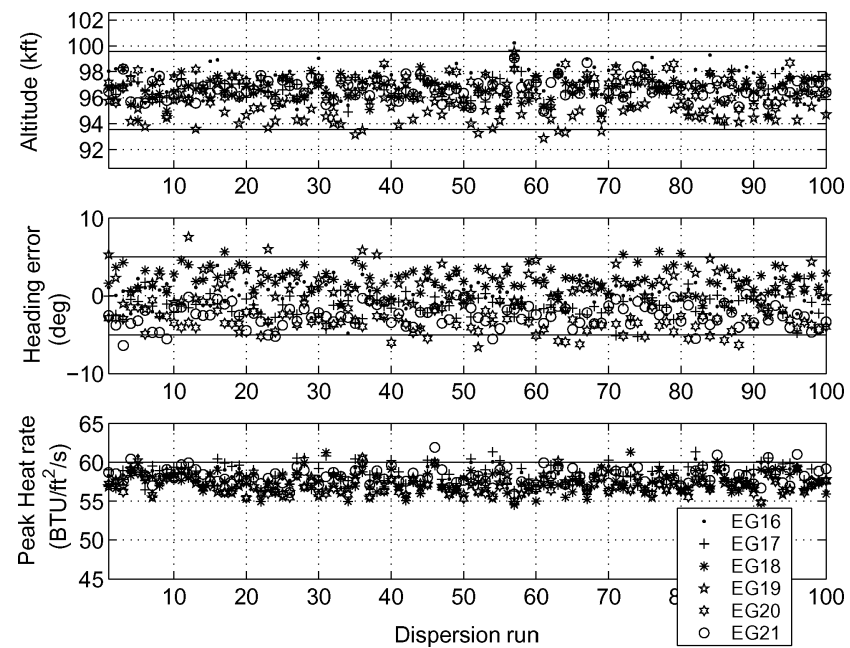

Fig. 11 Results of dispersion runs for EG16-EG21.

and 11, respectively. In Figs. 10 and 11, the dispersion run numbers are plotted on the abscissa. It can be seen in Figs. 10 and 11 that very few cases exceed the desirable 5-deg heading error and $3000-\mathrm{ft}$ altitude error limits, indicated by solid lines. No dispersion runs exceed the desirable peak heat rate limit of $75 \mathrm{Btu} /\left(\mathrm{ft}^{2} \cdot \mathrm{s}\right)$ for cases EG13-15, and only a few runs exceed the desirable $60 \mathrm{Btu} /\left(\mathrm{ft}^{2} \cdot \mathrm{s}\right)$ limit for cases EG16-21. All of the results fall within the allowable range, which is \pm 10 deg in heading, $\pm 6000 \mathrm{ft}$ in altitude, and below 65 and $80 \mathrm{Btu} /\left(\mathrm{ft}^{2} \cdot \mathrm{s}\right)$ peak heat rates for cases EG13-15 and EG16-21, respectively. The allowable tolerances for the other variables are presented in the following paragraph.

To rate and compare the performance of entry guidance algorithms quantitatively, a scoring procedure has been developed for the AGC study. ${ }^{19}$ Table 2 presents the criteria used for scoring the EG13-15 test cases. The first column contains the name of the flight parameter whose values are used for scoring. The second column gives a nominal value of the parameter (if applicable). The third and the fourth columns present the criteria for scores of 0 and 1 . If the value of a flight parameter lies within the ranges in columns 3 and 4, a linearly interpolated score is generated. (For example, if "Range to HAC at TAEM" is 25.5 or $34.5 \mathrm{n}$ mile, the score for this parameter is 0.5.) Each parameter score has a different weighting as presented in column 5 , and so range to $\mathrm{HAC}$ at TAEM has the highest weighting, whereas, "Max $\bar{q}-\alpha$ " has the lowest weighting. The scores for each parameter are averaged across the 100 dispersion cases. The weighted sum of these averages is then the total score for the test case. A very similar scoring table has been defined ${ }^{19}$ for the EG16-EG21 test cases; the flight parameters used for evaluation are the same, but the scoring weights are slightly different than those for the EG13-EG15 cases. EAGLE has scored very well in all of the test cases. The scores (out of a maximum of one) are 0.9808, 0.9995, 0.9980, 0.9942,
0.9941, 0.9979, 0.9798, 0.9967, and 0.9945 for cases EG13-EG21, respectively.

\section{Conclusions}

An entry guidance algorithm has been described, and its performance has been assessed for nine nominal return-from-orbit cases for a modified X-33 vehicle model in a high-fidelity simulation testbed. For each nominal case, 100 dispersion runs have also been simulated to assess the algorithm's ability to handle modeling and sensor errors, actuator degradation, and a range of initial conditions. The results of these simulations show that the entry guidance algorithm achieves the desired entry performance for almost all of the cases and the allowable entry performance for all of the cases as these requirements have been defined for an advanced guidance and control study described in the paper. In particular, the ability of the algorithm to guide large cross-range entries successfully has been demonstrated.

\section{Acknowledgment}

This research was funded by NASA Marshall Space Flight Center and by Universal Space Lines, LLC.

\section{References}

${ }^{1}$ Wingrove, R. C., "Survey of Atmosphere Reentry Guidance and Control Methods," AIAA Journal, Vol. 1, No. 9, 1963, pp. 2019-2029.

${ }^{2}$ Bryson, A. E., Jr., Carroll, F. J., Zvara, J., Black, A., Blatt, P., Bohling, R., Burke, M., Kanter, J., Lickly, D., Morth, R., Rosamond, D., and Wingrove, R., "Guidance and Navigation for Entry Vehicles," NASA SP-8015, Nov. 1968.

${ }^{3}$ Harpold, J. C., and Graves, C. A., Jr., "Shuttle Entry Guidance," Journal of the Astronautical Sciences, Vol. 27. No. 3, 1979 , pp. 239-268.

${ }^{4}$ Fuhry, D. P., "Adaptive Atmospheric Reentry Guidance for the Kistler K-1 Orbital Vehicle," Proceedings of the Guidance, Navigation, and Control Conference, AIAA, Reston, VA, 1999, pp. 1275-1288.

${ }^{5}$ Tu, K.-Y., Munir, M. S., Mease, K. D., and Bayard, D. S., "DragBased Predictive Tracking Guidance for Mars Precision Landing," Journal of Guidance, Control, and Dynamics. Vol. 23. No. 4, 2000. pp. $620-628$.

${ }^{6}$ Bryant, L., Tigges, M., and Ives, D., "Analytic Drag Control for Precision Landing and Aerocapture," AIAA Paper 98-4572, Aug. 1998.

${ }^{7}$ Carman, G., Ives, D., and Geller, D., "Apollo-Derived Mars Precision Lander Guidance,” AIAA Paper 98-4570, Aug. 1998.

${ }^{8}$ Powell, R. W., "Numerical Roll Reversal Predictor-Corrector Aerocapture and Precision Landing Guidance Algorithms for the Mars Surveyor Program 2001 Missions," AIAA Paper 98-4574, Aug. 1998.

${ }^{9}$ Roenneke, A. J., and Markl, A., "Reentry Control of a Drag vs Energy Profile," Journal of Guidance, Control, and Dynamics, Vol. 17, No. 5, 1994. pp. 916-920.

${ }^{10} \mathrm{Lu}$, P.,"Entry Guidance and Trajectory Control for Reusable Launch Vehicle," Journal of Guidance, Control, and Dynamics, Vol. 20, No. 1, 1997. pp. 143-149.

${ }^{11} \mathrm{Lu}, \mathrm{P} .$, and Hanson, J. M., "Entry Guidance for the X-33 Vehicle," Journal of Spacecraft and Rockets, Vol. 35, No. 3, 1998, pp. 342-349.

${ }^{12}$ Jouhaud, F., "Closed Loop Reentry Guidance Law of a Space Plane: Application to Hermes," Acta Astronautica, Vol. 26, No. 8-10, 1992, pp. 577-585.

${ }^{13}$ Powell, R. W., "Six-Degree-of-Freedom Guidance and Control Entry Analysis of the HL-20," Journal of Spacecraft and Rockets, Vol. 30. No. 5 , 1993, pp. 537-542.

${ }^{14}$ Mease, K. D., Chen, D. T., Teufel, P., and Schönenberger, H., "Reduced-Order Entry Trajectory Planning for Acceleration Guidance," Journal of Guidance, Control, and Dynamics, Vol. 25, No. 2, 2002. pp. 257-266.

${ }^{15}$ Mease, K. D., and Kremer, J.-P., "Shuttle Entry Guidance Revisited Using Nonlinear Geometric Methods," Journal of Guidance. Control, and Dynamics, Vol. 17, No. 6, 1994, pp. 1350-1356

${ }^{16}$ Dukeman, G. A.,"Profile-Following Entry Guidance Using Linear Quadratic Regulator Theory," AIAA Paper 2002-4457, Aug. 2002.

${ }^{17}$ Zimmerman, C., Dukeman, G. A., and Hanson, J. M., "Automated Method to Compute Orbital Reentry Trajectories with Heating Constraints," Journal of Guidance, Control, and Dynamics, Vol. 26, No. 4, 2003 , pp. 523-529. 
${ }^{18}$ Shen, Z., and Lu, P., "Onboard Generation of Three-Dimensional Constrained Entry Trajectories," Journal of Guidance, Control, and Dynamics, Vol. 26, No. 1, 2003, pp. 111-121.

${ }^{19}$ Hanson, J., and Jones, R., "Advanced Guidance and Control Methods for Reusable Launch Vehicles: Test Results," AIAA Paper 2002-4561, Aug. 2002.

${ }^{20}$ Hanson, J. M., "Advanced Guidance and Control Project for Reusable Launch Vehicles," AIAA Paper 2000-3957, Aug. 2000.

${ }^{21}$ Stengel, R. F., "Optimal Guidance for the Space Shuttle Transition," Journal of Spacecraft and Rockets. Vol. 11. No. 3. 1974. pp. 173-179.

${ }^{22}$ Vinh, N. X., Optimal Trajectories in Atmospheric Flight, Elsevier, New
York, 1981 pp. 26, 27.

${ }^{23}$ Hill, A. D., Anderson, D. M., Coughlin, D. J., and Chowdhry, R. S., “X-33 Trajectory Optimization and Design," AIAA Paper 98-4408, Aug. 1998.

${ }^{24}$ Hanson, J. M., Coughlin, D. J., Dukeman, G. A., Mulqueen, J. A., and McCarter, J. W., "Ascent, Transition, Entry, and Abort Guidance Algorithm Design for the X-33 Vehicle," AIAA Paper 98-4409, Aug. 1998.

D. B. Spencer Associate Editor 